\title{
Prospective ultrasonographic study of blood flow and testicular volume in patients submitted to surgical repair of inguinal hernia without using prosthesis ${ }^{1}$
}

\author{
Cláudio de Saboya David', Alberto Goldenberg"
}

'Fellow Master degree, Postgraduate Program in Interdisciplinary Surgical Science, Universidade Federal de São Paulo (UNIFESP), Brazil. Acquisition, analysis and interpretation of data; manuscript writing.

"Full Professor, Surgical Gastroenterology Division, Surgery Department, UNIFESP, Sao Paulo, Brazil. Critical revision, supervised all phases of the study.

\begin{abstract}
Purpose: To evaluate the behavior of arterial circulation and testicular volume in patients submitted to conventional inguinal hernia repair without the use of a synthetic prosthesis to reinforce the posterior wall.

Methods: A prospective observational clinical trial was performed on 26 male patients with unilateral inguinal hernia types I and II by the Nyhus classification, who underwent surgical correction using the modified Bassini technique. Bilateral Doppler ultrasonography was performed preoperatively, at the third and at the sixth postoperative month. The studied variables were: systolic peak velocity (SPV), diastolic peak velocity (DPV), resistance index (RI), pulsatility index (PI) and testicular volume.

Results: There were no statistically significant changes over time in the variables studied on the operated side: SPV ( $p=0.916)$, DPV $(p=0.304), \operatorname{RI}(p=0.879), \operatorname{PI}(p=0.475)$, and testicular volume $(p=0.100)$. The variables on the control side also did not change statistically until the sixth postoperative month: SPV $(p=0.784)$, DPV $(p=0.446), \mathrm{RI}(p=0.672), \mathrm{PI}(p=0.607)$, and testicular volume $(p=0.413)$.

Conclusion: Surgical correction of the inguinal hernia without the use of a prosthesis does not cause alterations in vascularization and testicular volume in the first six months postoperatively.

Key words: Hernia, Inguinal. Regional Blood Flow. Testis. Ultrasonography, Doppler.
\end{abstract}




\section{Introduction}

Inguinal hernia is a condition that affects the population with great frequency. According to a National Center for Health Statistics study, approximately 800.000 inguinal hernias are operated on a yearly basis in the United States.

Among the different techniques of surgical approach to inguinal hernia, a relevant benchmark came with the introduction of synthetic prostheses in the 1990s, with a direct impact on the results and consequently on patient satisfaction. Considering the simultaneous repair of more than one hernia in the same patient, it is reported that in the United States more than one million prostheses are implanted every year. The tension-free technique with mesh implants dramatically reduced recurrence rates in all types of hernias when compared to traditional repairs through sutures and tissue overlap. The technique also allows the reconstruction of large defects of the abdominal wall, previously considered untreatable ${ }^{1}$.

If, on the one hand, the repair of defects of the abdominal wall is one of the most common procedures performed by the general surgeon and the use of a prosthesis for correction is a safe procedure, its use is not without complications: hematomas and seromas may be present ${ }^{2}$, as well as recurrence and infection ${ }^{3}$. The incorporation with the surrounding tissues and the adhesions are directly related to the materials used ${ }^{4}$. As a complication, chronic pain may be incapacitating and may even require surgical approach ${ }^{5}$.

The adhesion caused by the mesh as well as infertility cases that followed its implantation, opened a wide field of research: Goldenberg $^{6}$ implanted a mesh unilaterally in 10 dogs and demonstrated focally in the testicle a decrease in spermatogenesis and degenerative process in $20 \%$ of the animals.
In the epididymis, chronic inflammation and dilation of the seminiferous tubules were observed in $70 \%$. In the vas deferens, a chronic inflammatory process was observed in $60 \%$ of the dogs. No histological changes were found on the contralateral side. Protasov et al. ${ }^{7}$ when studying rats, observed thickening of the mucosa and the wall of the vas deferens with mesh implants and found association with infertility.

It is not always possible to establish exactly the limits between the adverse effects caused by the mesh or the surgical manipulation. The study of testicular vascularization is an area of interest in the surgical correction of inguinal hernia. Among the reported complications resulting from surgical treatment, the evaluation of infertility after the use of prosthesis requires studies about testicular vascularization and function, as well as the assessment of the ductility of the vas deferens, that is, dysfunctions in postsurgical fertility in the correction of inguinal hernias are a fact well reported in the literature and are based basically on two pillars: vascular lesion with repercussions on the testis and lesion of the vas deferens.

According to Lima Neto et al. ${ }^{8}$, there are no changes in testicle size or testicular arterial flow with the use of a mesh in the correction of inguinal hernia. No studies were found on possible changes in testicular size and arterial flow in patients submitted to hernia repair without prosthesis, especially when considering interventions performed with the simple treatment of the hernia sac and interventions with posterior wall reinforcement without implantation of prosthesis, therefore, with only surgical manipulation when prosthetic material is not implanted.

Palabiyik et al. ${ }^{9}$, evaluated the effect of surgical manipulation on testicular perfusion using Doppler ultrasonography in inguinal hernia operations in pediatric patients (without 
the use of a prosthesis). They concluded that surgical manipulation performed in inguinal hernia operations in children causes transient alterations in the vascularization of the testicles in the immediate postoperative period, but return to normal in the late postoperative period.

Overall, the literature review shows a correlation between the inflammatory process caused by the mesh and the repercussion on the vas deferens and contiguous structures. At the same time, the circulatory changes when present appear to be transient after the application of prosthetic material.

Could the surgical manipulation of the inguinal region without the use of a foreign body cause regional inflammatory reaction to interfere with testicular vascularization and be detected by Doppler ultrasonography? Were adverse effects being attributed to the prosthesis, whereas, in fact, they would be from the surgical manipulation?

Therefore, this study aims to evaluate the arterial flow and testicular volume in patients submitted to surgical repair of inguinal hernia without prosthesis.

\section{Methods}

The clinical trial protocol was approved by the Research Ethics Committee of UNIFESP (no 1544/05).

We prospectively studied 26 patients from Hospital de Força Aérea do Galeão, Rio de Janeiro, from March 2006 to January 2008.

Initially, 42 patients were assigned, but 16 were excluded from the study. Three patients presented complications at the operative site, while four did not adequately attend the postoperative evaluation. In addition, nine patients were excluded during surgery, when it was found, during the dissection, that they did not have Nyhus I or II hernias, thus requiring the use of prostheses. Twenty-six patients remained in the study.

In all patients, preoperative evaluations and color Doppler examinations were performed at Hospital de Força Aérea do Galeão by the same operator. Surgical interventions were performed by the same surgeon, with the same surgical and anesthetic technique being strictly followed, although the anesthesiologist was not the same in all cases, but everyone knew that it was a scientific study and, therefore, employed the same technical rigor. The characteristics of the sample are shown in Table 1:

Table 1 - Sample characteristics.

\begin{tabular}{|c|c|}
\hline Sample & $(n=26)$ \\
\hline $\begin{array}{l}\text { Age (years) } \\
\text { Mean } \\
\text { Minimum - maximum }\end{array}$ & $\begin{array}{l}20.46 \\
16-35\end{array}$ \\
\hline $\begin{array}{l}\text { Hernia side } \\
\text { Right } \\
\text { Left }\end{array}$ & $\begin{array}{l}18 \\
8\end{array}$ \\
\hline $\begin{array}{l}\text { Surgical Intervention } \\
\text { Hospital de Força Aérea do } \\
\text { Galeão }\end{array}$ & 26 \\
\hline
\end{tabular}

Inclusion criteria were male patients, aged between 16 and 33 years, and patients with unilateral inguinal hernia - Nyhus I and II. Patients with femoral hernia, bilateral hernia, other associated hernias, testicular diseases, previous surgery on the inguinal region, varicocele, incarcerated or strangulated hernia, previous surgery on the testicle, scrotum or prostate were excluded. Also excluded were patients who underwent pelvic radiotherapy, patients with diabetes, hypertension, chronic or venous arterial disease, past abscess in the inguinal region, cognitive alterations and psychiatric disorders, as well as complications occurring at the operative site such as hematoma, seroma or infection.

Doppler Ultrasonography was performed preoperatively and postoperatively 
on both the operated side and the contralateral side (control), according to Figure 1.

The systolic peak velocity (SPV), diastolic peak velocity (DPV), resistance index (RI), and pulsatility index (PI), in addition to the testicular volume (TV) were assessed on both sides. The trans-testicular artery was identified (Figure 2), the testicle parenchymal flow was assessed (Figure 3), and the testicular artery was identified one centimeter from the upper pole of the testis with the transducer angled at 60 degrees, when waveforms compatible with the arterial flow were obtained (Figure 4). The testicular volume was measured by multiplying the 3 dimensions of the largest axes in $\mathrm{cm}$, whose result was later multiplied by a constant 0.52 (Figure 5).

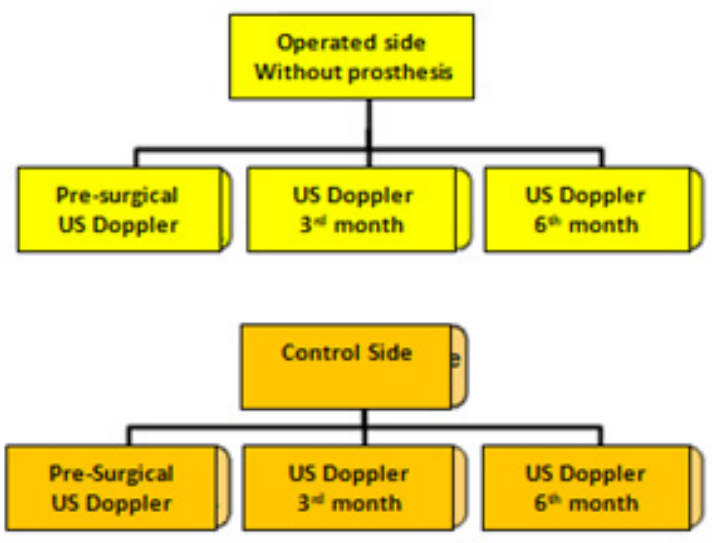

Figure 1 - Study design

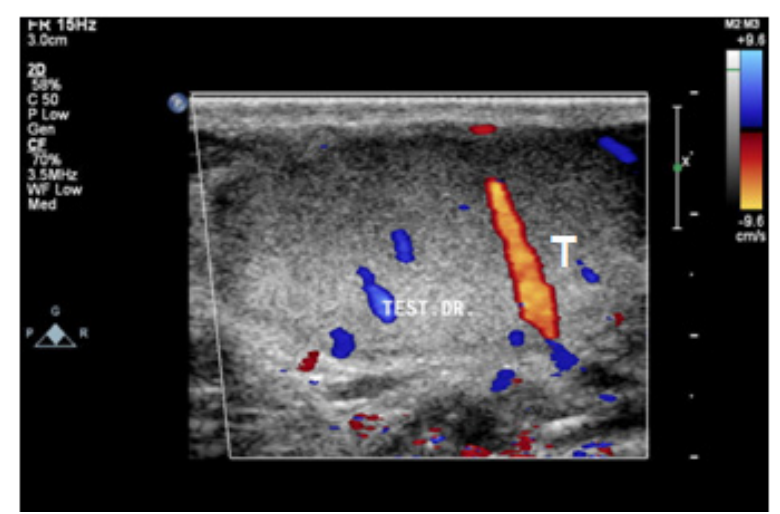

Figure 2 - Identification of the trans-testicular artery $(\mathrm{T})$.

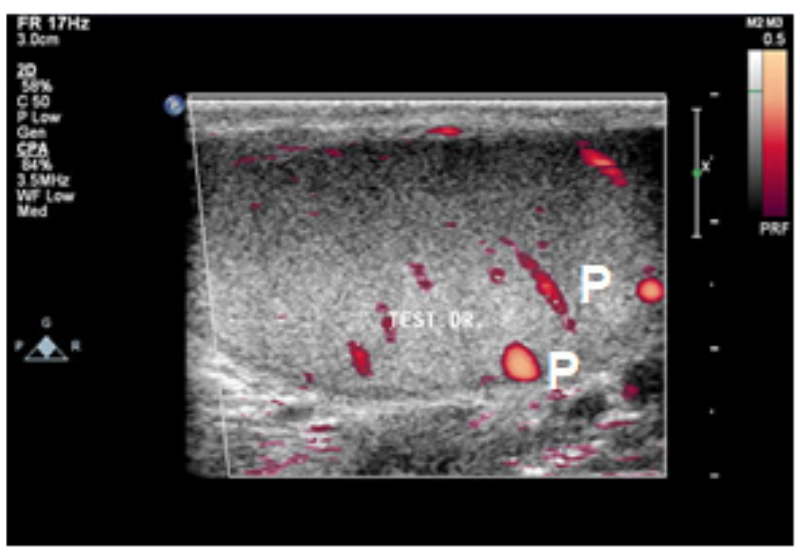

Figure 3 - Evaluation of perfusion of the testicular parenchyma $(\mathrm{P})$.

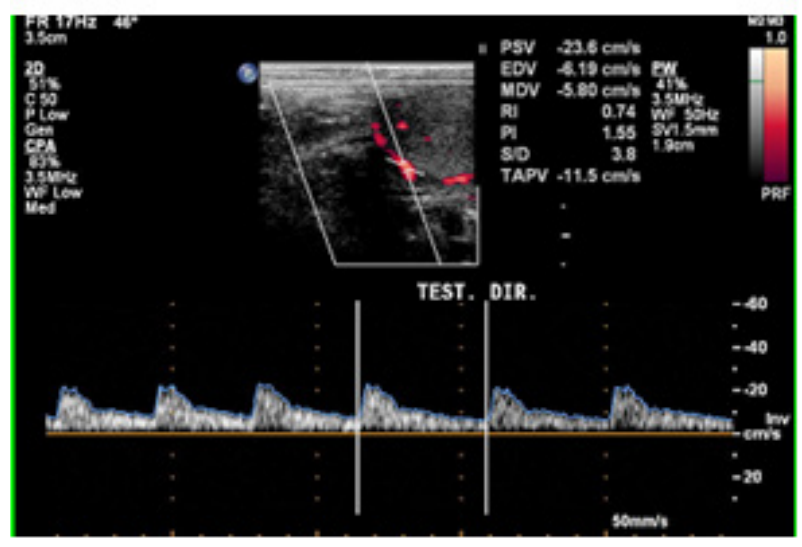

Figure 4 - The testicular artery was identified in its distal portion $1 \mathrm{~cm}$ from the upper pole of the testis, using a $60^{\circ}$ angulation transducer with the patient in the supine position.

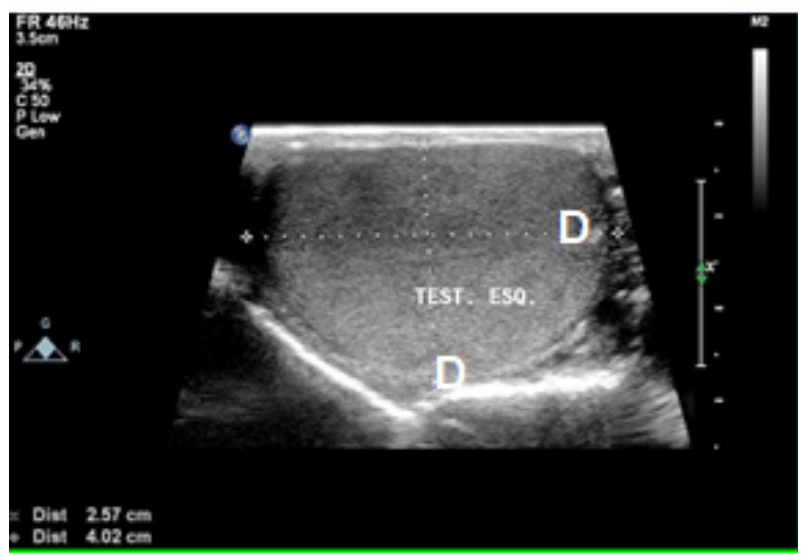

Figure 5 - Testicular volume was obtained by multiplying the three dimensions in $\mathrm{cm}(D)$ by a constant (0.52). 
Systolic peak velocity (SPV) was considered to be the rate of blood flow in the systole at the center of the artery assessed in $\mathrm{cm} / \mathrm{s}$ and the Diastolic Peak Velocity (DPV), the velocity of blood flow in the diastole at the center of the artery, also expressed in $\mathrm{cm} / \mathrm{s}$.

The resistance index (RI) was obtained by subtracting the Systolic Peak Velocity by the Diastolic Peak Velocity and the result divided by the Systolic Peak Velocity (RI=SPV-DPV/SPV).

The mean velocity (MV) was calculated by dividing the Systolic Peak Velocity by the Diastolic Peak Velocity (MV = SPV / DPV).

The Pulsatility Index (PI) was defined by the subtraction of the Systolic Peak Velocity by the Diastolic Peak Velocity and the result divided by the Mean Velocity (PI = SPV-DPV/ MV).

\section{Surgical considerations}

- Epidural anesthesia

- Access in the inguinal region through a $5-\mathrm{cm}$ oblique incision from the bisector of the angle formed by the lateral border of the rectus abdominis muscle and the inguinal ligament as a reference point.

- Dieresis in planes.

- Access to the inguinal canal with identification and preservation of its elements.

- Treatment of the indirect hernia sac (Figure 6A).

- Framing in the Nyhus Classification.

- Reinforcement of the rear wall as per Modified Bassini (Figure 6B).
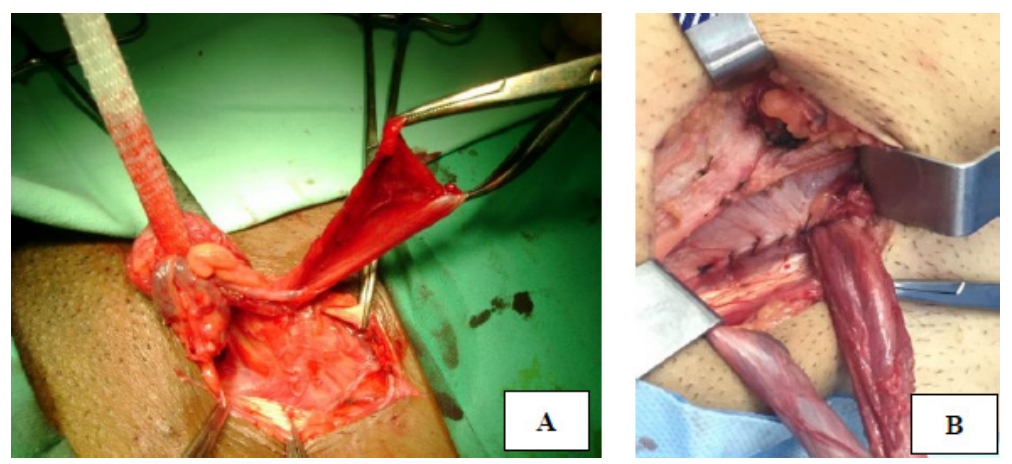

Figure 6 - A. The indirect hernia sac. B. Reinforcement of the rear wall as per modified Bassini.

\section{Statistical analysis}

The data obtained in this study were analyzed using:

a) Tables, arithmetic means, medians, standard deviations, minimum and maximum values;

b) Mann-Whitney non-parametric test, for comparison of the values of the Treated and Control groups for the variables under study, at the pre, 3-month and 6-month timepoints.

c) Friedman's non-parametric test, for comparing the pre, 3-month and 6-month timepoints, for the variables selected for the study.
In the application of the statistical tests, a level of significance of $5 \%$ of probability $(P<0.05)$ was used to verify statistically significant differences.

The SPSS Software was used to perform data analysis.

\section{- Results \\ There is no significant difference between the pre, 3-month and 6-month timepoints in relation to the systolic velocity, on the operated and control sides (Table 2).}


Table 2 - Arithmetic means, standard deviations, medians, minimum and maximum values, comparison of the PRE, 3-MONTH AND 6-MONTH timepoints of the Treatment and Control groups, according to the systolic velocity.

\begin{tabular}{|c|c|c|c|c|c|}
\hline \multicolumn{6}{|c|}{ SYSTOLIC VELOCITY - OPERATED SIDE } \\
\hline Assessments & Pre & 3-month & 6-month & Test & Significance \\
\hline Arithmetic mean & 20.03 & 20.12 & 20.19 & & \\
\hline Standard deviation & 7.60 & 7.87 & 7.25 & $X r^{2}=0.175$ & $(P=0.916)^{n . s}$ \\
\hline Median & 18.45 & 17.60 & 18.57 & & \\
\hline Minimum & 7.20 & 7.21 & 6.54 & & \\
\hline Maximum & 36.32 & 36.54 & 37.22 & & \\
\hline \multicolumn{6}{|c|}{ SYSTOLIC VELOCITY - CONTROL SIDE } \\
\hline Assessments & Pre & 3-month & 6-month & Test & Significance \\
\hline Arithmetic mean & 19.18 & 19.23 & 18.96 & & \\
\hline Standard deviation & 6.62 & 6.24 & 5.81 & $X r^{2}=0.485$ & $(P=0.784)^{n . s}$ \\
\hline Median & 18.04 & 17.44 & 17.32 & & \\
\hline Minimum & 8.42 & 9.17 & 9.79 & & \\
\hline Maximum & 38.16 & 36.45 & 31.55 & & \\
\hline
\end{tabular}

n.s=non significant

Figure 7 shows the time course of systolic velocity on both sides.

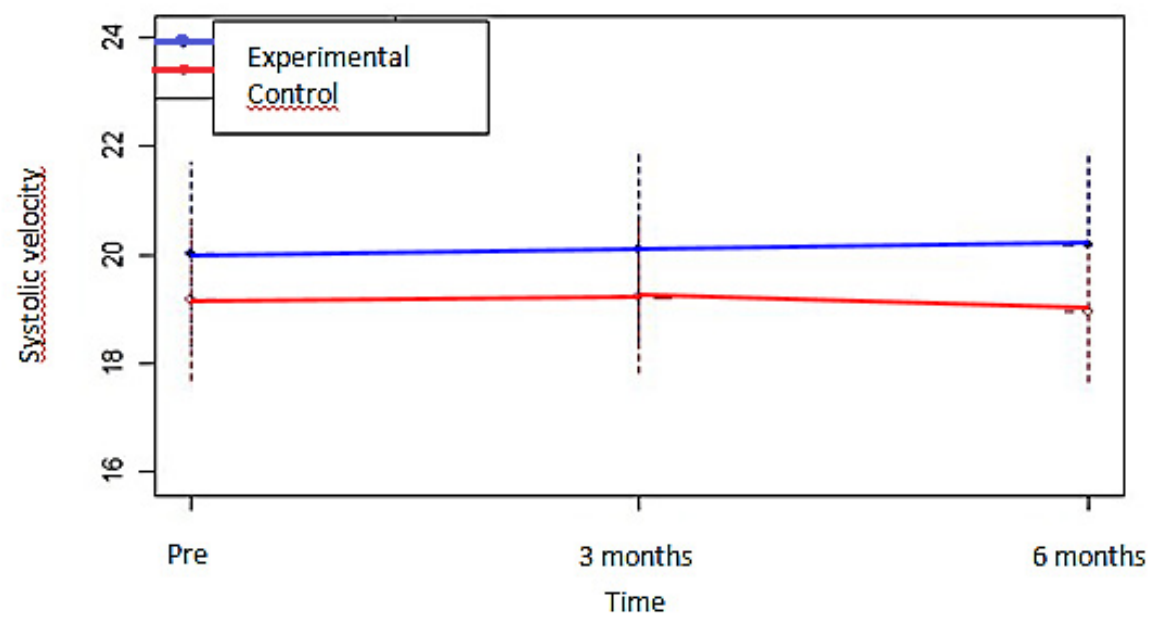

Figure 7 - Mean profiles of the systolic velocity on each side, over time. 
There is no significant difference between the pre, 3-month and 6-month timepoints in there There is no significant difference between the pre, 3-month and 6-month timepoints in relation to the diastolic velocity on the operated and control sides (Table 3).

Table 3 - Arithmetic means, standard deviations, medians, minimum and maximum values, comparison of the PRE, 3-MONTH and 6-MONTH timepoints of the Treatment and Control groups, according to the diastolic velocity.

\begin{tabular}{|c|c|c|c|c|c|}
\hline \multicolumn{6}{|c|}{ DIASTOLIC VELOCITY - OPERATED SIDE } \\
\hline Assessments & Pre & 3-month & 6-month & Test & Significance \\
\hline Arithmetic mean & 3.82 & 3.66 & 3.61 & & \\
\hline Standard deviation & 2.07 & 2.28 & 2.04 & $\mathrm{Xr}^{2}=2.38$ & $(P=0.304)^{n . s}$ \\
\hline Median & 4.07 & 3.98 & 3.35 & & \\
\hline Minimum & 0.39 & 0.15 & 0.35 & & \\
\hline Maximum & 8.25 & 9.92 & 7.65 & & \\
\hline \multicolumn{6}{|c|}{ DIASTOLIC VELOCITY - CONTROL SIDE } \\
\hline Assessments & Pre & 3-month & 6-month & Test & Significance \\
\hline Arithmetic Mean & 3.96 & 4.03 & 3.75 & & \\
\hline Standard deviation & 1.54 & 1.49 & 1.19 & $X r^{2}=1.61$ & $(P=0.446)^{n . s}$ \\
\hline Median & 3.54 & 3.87 & 3.53 & & \\
\hline Minimum & 1.97 & 1.48 & 2.15 & & \\
\hline Maximum & 7.98 & 7.41 & 7.14 & & \\
\hline
\end{tabular}

Figure 8 shows the temporal evolution of the diastolic velocity on both sides.

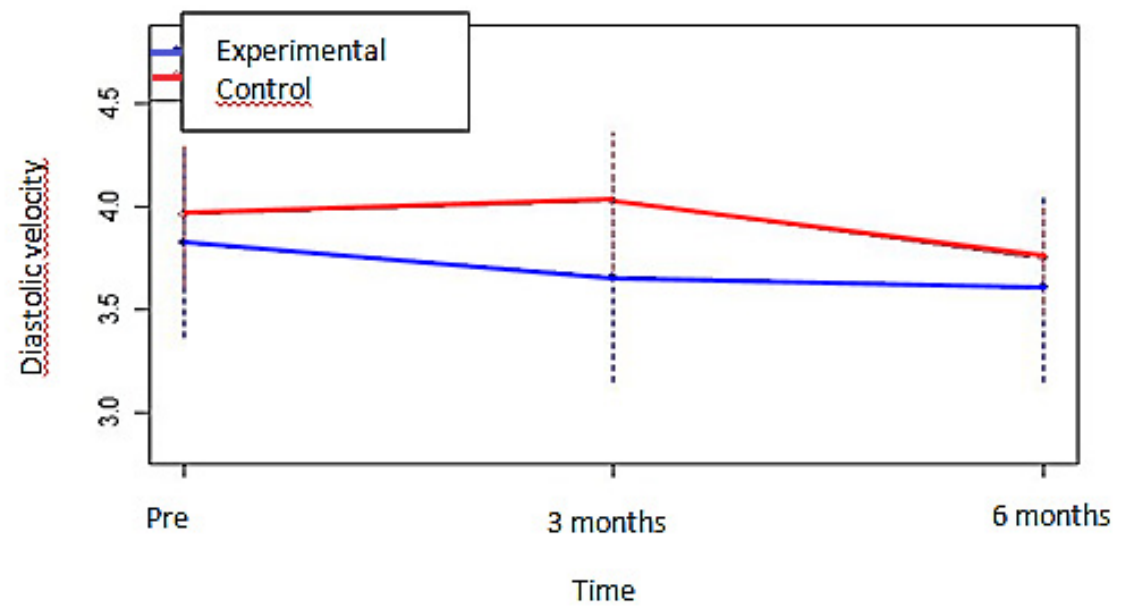

Figure 8 - Mean profiles of diastolic velocity on each side, over time. 
There is no significant difference between the pre, 3-month and 6-month timepoints in relation to the resistance index, on the operated and control sides (Table 4).

Table 4 - Arithmetic means, standard deviations, medians, minimum and maximum values, comparison of the PRE, 3-MONTH and 6-MONTH timepoints of the Treatment and Control groups, according to the resistance index.

\begin{tabular}{|c|c|c|c|c|c|}
\hline \multicolumn{6}{|c|}{ RESISTANCE INDEX - OPERATED SIDE } \\
\hline Assessments & Pre & 3-month & 6-month & Test & Significance \\
\hline Arithmetic mean & 0.79 & 0.81 & 0.80 & & \\
\hline Standard deviation & 0.12 & 0.11 & 0.11 & $X r^{2}=0.26$ & $(P=0.879)^{n . s}$ \\
\hline Median & 0.81 & 0.82 & 0.80 & & \\
\hline Minimum & 0.56 & 0.62 & 0.58 & & \\
\hline Maximum & 0.98 & 0.99 & 0.97 & & \\
\hline \multicolumn{6}{|c|}{ RESISTANCE INDEX - CONTROL SIDE } \\
\hline Assessments & Pre & 3-month & 6-month & Test & Significance \\
\hline Arithmetic mean & 0.77 & 0.77 & 0.78 & & \\
\hline Standard deviation & 0.10 & 0.09 & 0.09 & $\mathrm{Xr}^{2}=0.80$ & $(P=0.672)^{n . s}$ \\
\hline Median & 0.80 & 0.79 & 0.81 & & \\
\hline Minimum & 0.50 & 0.56 & 0.57 & & \\
\hline Maximum & 0.94 & 0.92 & 0.91 & & \\
\hline
\end{tabular}

n.s=non significant

Figure 9 shows the time evolution of the resistance index on both sides.

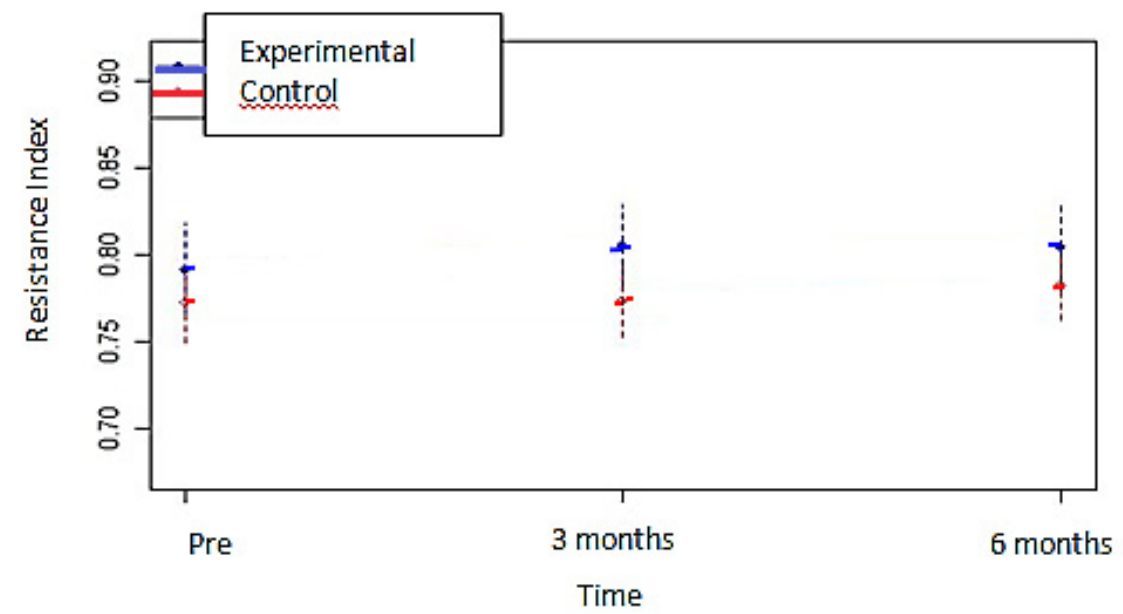

Figure 9 - Average profiles of resistance index on each side, over time. 
Table 5 also shows no significant difference between the pre, 3-month and 6-month timepoints in relation to the pulsatility index on the operated side or on the control side.

Table 5 - Arithmetic means, standard deviations, medians, minimum and maximum values, comparison of the PRE, 3-MONTH and 6-MONTH timepoints of the Treatment and Control groups, according to the pulsatility index.

\begin{tabular}{|c|c|c|c|c|c|}
\hline \multicolumn{6}{|c|}{ PULSATILITY INDEX - OPERATED SIDE } \\
\hline Assessments & Pre & 3 months & 6 months & Test & Significance \\
\hline Arithmetic mean & 2.86 & 2.77 & 2.72 & & \\
\hline Standard deviation & 1.33 & 1.48 & 1.29 & $X r^{2}=1.49$ & $(P=0.475)^{n . s}$ \\
\hline Median & 2.92 & 2.90 & 2.78 & & \\
\hline Minimum & 0.38 & 0.15 & 0.34 & & \\
\hline Maximum & 5.58 & 6.43 & 5.46 & & \\
\hline \multicolumn{6}{|c|}{ PULSATILITY INDEX - CONTROL SIDE } \\
\hline Assessments & Pre & 3-month & 6-month & Test & Significance \\
\hline Arithmetic mean & 2.97 & 3.04 & 2.88 & & \\
\hline Standard deviation & 0.89 & 0.92 & 0.65 & $X r^{2}=1.0$ & $(P=0.607)^{n . s}$ \\
\hline Median & 2.82 & 2.92 & 2.73 & & \\
\hline Minimum & 1.77 & 1.35 & 1.86 & & \\
\hline Maximum & 4.75 & 4.63 & 4.34 & & \\
\hline
\end{tabular}

n.s=non significant

Figure 10 shows the temporal evolution of pulsatility on both sides.

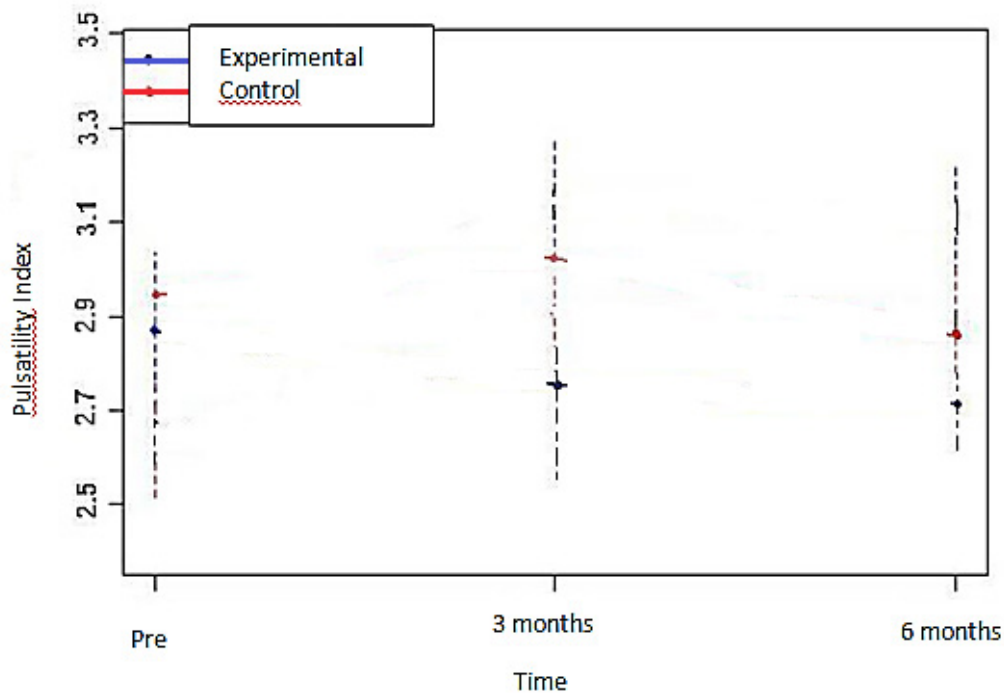

Figure 10 - Average profiles of the pulsatility index on each side over time. 
Table 6 shows no significant difference between the pre, 3-month and 6-month timepoints in relation to the testicular volume, on the operated and control sides.

Table 6 - Arithmetic means, standard deviations, medians, minimum and maximum values, comparison of the PRE, 3-MONTH and 6-MONTH timepoints of the Treatment and Control groups, according to the testicular volume.

\begin{tabular}{|c|c|c|c|c|c|}
\hline \multicolumn{6}{|c|}{ TESTICULAR VOLUME - OPERATED SIDE } \\
\hline Assessments & Pre & 3-month & 6-month & Test & Significance \\
\hline Arithmetic mean & 14.20 & 14.74 & 15.38 & & \\
\hline Standard deviation & 3.93 & 4.45 & 4.26 & $X r^{2}=4.60$ & $(P=0.100)^{n . s}$ \\
\hline Median & 14.01 & 14.87 & 15.52 & & \\
\hline Minimum & 7.80 & 8.40 & 8.0 & & \\
\hline Maximum & 21.40 & 24.97 & 23.70 & & \\
\hline \multicolumn{6}{|c|}{ TESTICULAR VOLUME - CONTROL SIDE } \\
\hline Assessments & Pre & 3-month & 6-month & Test & Significance \\
\hline Arithmetic mean & 14.86 & 14.84 & 15.41 & & \\
\hline Standard deviation & 4.22 & 4.31 & 4.16 & $X r^{2}=1.77$ & $(P=0.413)^{n . s}$ \\
\hline Median & 14.87 & 14.10 & 15.76 & & \\
\hline Minimum & 9.48 & 8.52 & 8.47 & & \\
\hline Maximum & 26.80 & 24.21 & 22.57 & & \\
\hline
\end{tabular}

n.s=non significant

Figure 11 shows the temporal evolution of testicular volume on both sides.

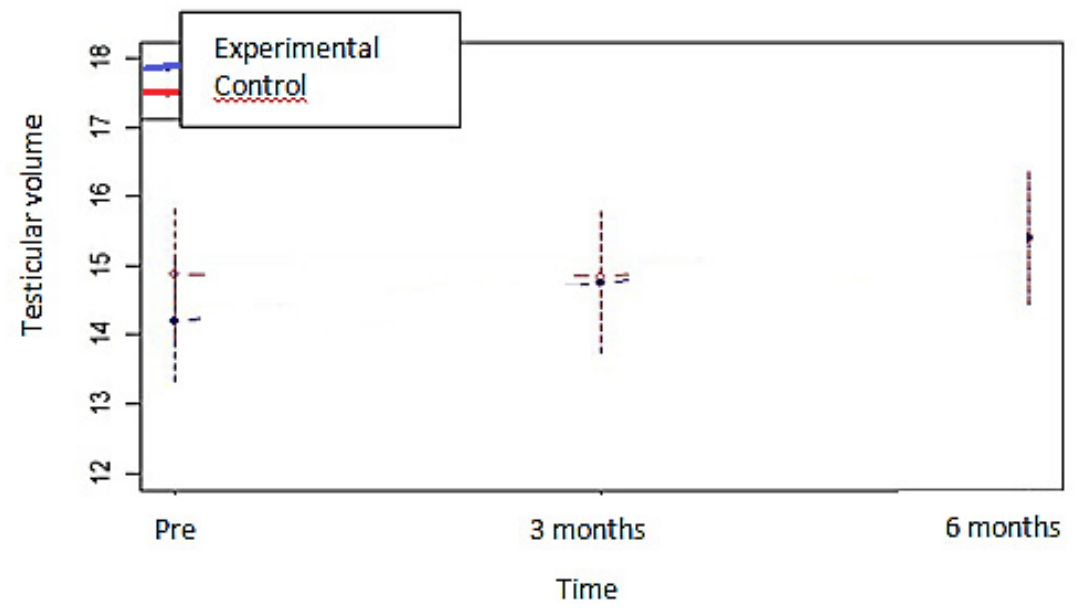

Figure 11 - Mean profiles of testicular volume on each side over time. 


\section{- Discussion}

Undoubtedly, the use of prosthesis in the inguinal hernias reduced the relapse rates in 10 to $15 \%$ with the Bassini technique and variants to less than $5 \%$ after the implantation of the prostheses ${ }^{10}$. However, almost all studies dealing with recurrence do not consider in isolation the different types and classifications of inguinal hernias. The tendency to relapse an indirect inguinal hernia that has been operated only with hernia sac treatment may be different from a direct hernia with destruction of the posterior wall of the inguinal canal. At first, there is no scientific justification for treating different defects with the same operative technique.

The classification schemes for the repair of hernias are important instruments of standardization in the dissemination of these results. They allowed a standardization in the registry of hernias of the inguinal region, enabling the scientific communication and development of works, selecting groups of homogenous patients. The Nyhus classification allowed for uniformity in the approach of the inguinal-femoral hernias ${ }^{11}$. It takes into account the dimensions of the internal inguinal ring and the state of the posterior wall of the inguinal canal that are verified during surgery. The classification proposed by Gilbert in 1986 is based on the anatomical and functional defects observed in the intraoperative period.

Operating inguinal hernia those days without using a prosthesis leads many surgeons to think that the patient will be harmed, since the use of the mesh in addition to being widespread, is currently considered by many surgeons as an indispensable material for the surgical procedure. However, when considering its possible deleterious effects, it is observed concomitantly that there is scientific justification in the literature for not applying mesh in all cases of inguinal hernia.
In the selected cases, there are low rates of relapse without the use of a prosthesis, and it is possible to treat different defects with appropriate techniques for each case.

There are other justifications for not using mesh in all cases of inguinal hernia repair. Haastrup et al. ${ }^{12}$ investigated the rates of reoperation after repair of indirect inguinal hernia by conventional technique with and without prosthesis in young male patients and in other age groups. Based on the finding that younger patientsareatgreater risk of developing chronic pain after conventional inguinal hernia repair with prostheses, compared with older patients, prosthesis avoidance in young patients may be justified, provided there is an acceptable risk of recurrence in comparison with other groups and with the Lichtenstein repair. The reoperation rate for the group of young patients aged 18-29 years without prosthesis was significantly lower compared to all other age groups.

Even today hypotheses exist about the cause of damage to the vas deferens during surgeries for correction of inguinal hernia. Khodari et al. ${ }^{13}$ studied 69 patients with azoospermia and performed proximal surgical collection of sperm in three groups: one submitted to correction of bilateral hernia, one with risk factors for infertility and the third with unilateral hernia. They did not find a positive relation with the surgical technique used or even the use of a prosthesis. They concluded that the obstruction of the vas deferens after correction of inguinal hernia is a possible and underestimated iatrogenic event, but did not mention whether the obstruction of the duct was due to the operative technique (ligature) or loco-regional inflammatory reaction by surgical manipulation or prosthesis implantation.

Complications after correction of inguinal hernia were historically attributed to the surgery technique, blaming the inexperience of the surgeon. Adverse effects 
on the spermatic cord - testicular atrophy and occlusion of the vas deferens - are feared because the irritated patient, fearing his masculinity has been threatened, often resorts to legal suits. Testicular ischemia was attributed as a result of a very tight reconstruction of the internal inguinal ring. Lesions in the vas deferens may arise after inadvertent dissection of the hernia sac and may be ligated, sectioned, or devascularized. These lesions may be underestimated, since the majority of men submitted to hernia repair in the groin tend to be older, without concern for their reproductive capacity. It is added that most inguinal hernias are unilateral, which rarely causes sterility in younger patients. However, a significant minority has bilateral repairs. In addition, cryptorchidism or even epididymitis may eliminate the contralateral reproductive contribution ${ }^{14}$.

From the works of Goldenberg et al. ${ }^{6}$, and Goldenberg and De Paula ${ }^{15}$, it was clear that the inflammatory reaction provoked by the prosthesis extends to the spermatic cord and the vas deferens in much greater intensity when compared to the simple surgical manipulation without prosthesis implantation.

Nevertheless, the topics inguinal hernia and infertility are inserted in a larger universe than one could initially imagine, encompassing countless questions, with wide field for research. The purpose of this study, when studying the repercussions on testicular circulation caused by surgical manipulation without the use of prosthesis, is actually part of the clarification within a line of research that may in the future clarify the various aspects of the relationship between infertility and correction of inguinal hernias.

This study did not find changes in the circulatory variables measured over time after intervention without the use of prosthesis, complementing the results of Lima Neto et al. ${ }^{8}$, who conducted a study with prosthesis implantation. It should be noted, however, that the circulatory behavior in the presence of inguinal hernia and after its correction, does not present uniform results in the literature. Although there is some divergence, there is no marked discrepancy between the results to the point of labeling them as conflicting.

Tuncer et $a l^{16}$ studied the testicular arterial flow and volume after intervention on the inguinal region without the use of a mesh in 23 children and reported that patients with inguinal hernia, cord cysts and hydrocele on the right presented a decrease in the preoperative period of the testicular arterial flow with statistical significance, but this alteration was reversed after surgery. They attributed the decreased flow to the compression of the vessels of the spermatic cord, but did not explain why this alteration did not occur to the left. Supporting this, Ozdamar and Karakus ${ }^{17}$ recommended a conservative approach and Doppler ultrasonography follow-up in cases of testicular ischemia caused by compression due to incarcerated inguinal hernia in children, emphasizing that surgery should be performed as soon as possible for the reversal of the process.

The last two studies mentioned lead us to consider that testicular vascularization in children tends to return to normal patterns once the cause that determines their deviation has ceased. It remains to be seen whether the vascular recovery capacity is the same as in adults, which could lead, for example, to a review of the approach to cases of testicular torsion ischemia in children and young adults. This opens the door to further investigation.

Alterations are also found in the adult population and usually follow the pattern of postoperative reversibility. Bulus et al. ${ }^{18}$ found no adverse effects on testicular perfusion at the Doppler in patients submitted to hernia repair by the Lichtenstein technique. They found, however, a statistically significant increase 
in mean arterial flow velocity on the side of the hernia after surgery, and this change was considered transient.

If some studies have shown that the presence of inguinal hernia causes transient decrease of the testicular arterial flow, supposedly due to a mechanical effect, it is not well understood if this alteration is more pronounced in the indirect inguinal hernia that has an intrafunicular component or in the direct inguinal hernia. In a preliminary analysis of the literature, it appears that the hernial sac volume and, consequently, its compressive capacity, has a more significant effect. In another study, there was an increase in testicular flow in the postoperative period after correction of incarcerated inguinal hernia.

The US Doppler is a good test to measure the circulatory characteristics. Usually, in the adult individual, the testicles measure 3 to $5 \mathrm{~cm}$ in its longitudinal axis, 2 to $3 \mathrm{~cm}$ in the transverse axis and 1.5 to $2 \mathrm{~cm}$ in the anteroposterior axis. The testicular volume was obtained by multiplying the three major axes with each other and the result by the mathematical constant 0.52 , with the normal value in the adult being $15 \pm 8 \mathrm{~mL}$.

Systolic velocity (SPV) consists of the velocity of blood flow in systole at the center of the artery and diastolic velocity (DPV) is the velocity of blood flow in the diastole at the center of the artery.

Testicular volume is an important measure of testicular atrophy that may occur after inguinal hernia surgeries, and the testicular arterial flow is essential to maintain testicular volume and function.

The diastolic velocity is mentioned as an important parameter for the diagnosis of significant arterial occlusion. In stenosis where the vessel diameter is reduced to $70 \%$ or more, the DPV increases, but goes to zero when the occlusion is complete.
The pulsatility index is an important parameter for the evaluation of arterial stenosis. It is more meaningful for less caliber vessels.

The association of measured variables also has clinical applicability. Thus, the resistance index (RI) and the pulsatility index (PI) are inversely proportional to the testicular blood flow.

\section{Conclusion}

The surgical repair of inguinal hernia without the use of a prosthesis does not cause alterations in vascularization and testicular volume.

\section{References}

1. Cobb WS, Kercher KW, Heniford BT. The argumentforlightweightpolypropylenemesh in hernia repair. Surg Innov. 2005;12(1):639. doi: 10.1177/155335060501200109.

2. Wantz GE. Experience with the tension-free hernioplasty for primary inguinal hernias in men. J Am Coll Surg. 1996;183(4):351-6. PMID: 8843264.

3. Hasegawa S, Yoshikawa T, Yamamoto $Y$, Ishiwa N, Morinaga S, Noguchi $\mathrm{Y}$, Ito $\mathrm{H}$, Wada N, Inui K, Imada T, Rino Y, Takanashi Y. Long-term outcome after hernia repair with the prolene hernia system. Surg Today. 2006;36(12):1058-62. doi: 10.1007/s00595006-3311-9.

4. Novitsky YW, Harrell AG, Cristiano JA, Paton BL,Norton HJ, PeindIRD, Kercher KW, Heniford BT. Comparative evaluation of adhesion formation. strength of ingrowth. and textile properties of prosthetic meshes after longterm intra-abdominal implantation in a rabbit. J Surg Res. 2007;140(1):6-11. doi: 10.1016/j.jss.2006.09.015.

5. Palumbo P, Minicucci A, Nasti AG, Simonelli I, Vietri F, Angelici AM. Treatment for persistent chronic neuralgia after inguinal hernioplasty. Hernia 2007;11(6):527-31. doi: 10.1007/s10029-007-0268-6.

6. Goldenberg A, Matone J, Marcondes W, Focchi G. Efeitos da tela de polipropileno no 
Prospective ultrasonographic study of blood flow and testicular volume in patients submitted to surgical repair of inguinal hernia without using prosthesis David CS et al.

testículo, epidídimo e ducto deferentes de cães. Acta Cir Bras. 2001;16(4):226-30. doi: 10.1590/S0102-86502001000400007.

7. Protasov AV, Krivtsov GA, Mikhaleva LM, Tabulka AV, Shukhtin Nlu. Effects of inguinal hernioplasty mesh implant on reproductive function. Khirurgiia (Mosk). 2010;(8):28-32. PMID: 20823817.

8. Lima Neto EV, Goldenberg A, Jucá MJ. Prospective study on the effects of a polypropylene prosthesis on testicular volume and arterial flow in patients undergoing surgical correction for inguinal hernia. Acta Cir. Bras. 2007;22(4):266-71. PMID: 17625664.

9. Palabiyik FB, Cimilli T, Kayhan A, Toksoy N. Do the manipulations in pediatric inguinal hernia operations affect the vascularization of testes? J Pediatr Surg. 2009;44(4):788-90. doi: 10.1016/j.jpedsurg.2008.10.063.

10.Fitzgibbons RJ Jr. Can we be sure polypropylene mesh causes infertility? Ann Surg. 2005;241(4):559-61. PMID: 15798456.

11.Nyhus LM, Klein MS, Rogers FB. Inguinal hernia. Curr Probl Surg. 1991 Jun;28(6):40150. PMID: 2032462.

12. Haastrup E, Andresen K, Rosenberg J. Low reoperation rates in young males after sutured repair of indirect inguinal hernia: arguments for a tailored approach. Am J Surg. 2017 Nov;214(5):844-8. doi: 10.1016/j. amjsurg.2017.02.015.

13.Khodari M, Ouzzane A, Marcelli F, Yakoubi R, Mitchell V, Zerbib P, Rigot JM. Azoospermia and a history of inguinal hernia repair in adult. Prog Urol. 2015;25(12):692-7. doi: 10.1016/j.purol.2015.06.008.

14.Read RC. Damage to the spermatic cord from groin herniorrhaphy: a review. In: Schumpelick V, Fitzgibbons RJ. Hernia repair sequelae. Springer Berlin Heidelberg: 2010. p.71-5. doi: 10.1007/978-3-642-11541-7.

15.Goldenberg A, De Paula JF. Effects of the polypropylene mesh implanted through inguinotomy in the spermatic funiculus, epididium and testis of dogs. Acta Cir Bras. 2005;20(6):461-7. doi: /S010286502005000600012.

16. Tuncer AA, Peker T, Acar MB, Embleton DB, Cetinkursun S. A comparison of preoperative and postoperative testicular volume and blood flow in patients with inguinal hernia, hydrocele, and cord cyst: a prospective cohort study. Pak J Med Sci. 2017;33(2):3638. doi: 10.12669/pjms.332.12487.

17. Ozdamar MY, Karakus OZ. Testicular ischemia caused by incarcerated inguinal hernia in infants: incidence, conservative treatment procedure, and follow-up. Urol J. 2017;14(4):4030-3. PMID: 28670671.

18. Bulus H, Dogan M, Tas A, Agladioglu K, Coskun A. The effects of Lichtenstein tension-free mesh hernia repair on testicular arterial perfusion and sexual functions. Wien Klin Wochenschr. 2013;125(3-4):96-9. doi: 10.1007/s00508-013-0321-7.

\section{- Acknowledgements}

To Prof. Dr. Pedro Carvalho Rodrigues for statistical analysis, and Profa Mariela Palacios for English language.

\section{Correspondence:}

Cláudio de Saboya David

Avenida Prefeito Dulcídio Cardoso, 1200, BI 2/603

22620-311 Rio de Janeiro - RJ Brasil

Tel.: (55 21)99987-4726

csdavid@uol.com.br

Received: Nov 16, 2017

Review: Jan 18, 2018

Accepted: Feb 20, 2018
Conflict of interest: none

Financial source: none

\begin{abstract}
${ }^{1}$ Research performed at Hospital de Força Aérea do Galeão, Rio de Janeiro-RJ, Brazil. Part of Master degree thesis, Postgraduate Program in Interdisciplinary Surgical Science, UNIFESP. Tutor: Prof. Alberto Goldenberg.
\end{abstract}

